\title{
About the Crystallization of Abiotic Coded Matter
}

\author{
Benoît Éric Petit, Bernard Lotz* and Jean-François Lutz ${ }^{1 *}$ \\ ${ }^{1}$ Université de Strasbourg, CNRS, Institut Charles Sadron UPR22, 23 rue du Loess, 67034 Strasbourg Cedex 2, \\ France. Email: bernard.lotz@ics-cnrs.unistra.fr; jflutz@ unistra.fr. ${ }^{2}$
}

\section{A. Experimental procedures}

\section{A.1. Materials}

The digitally-encoded oligourethanes were synthesized by orthogonal solid-phase synthesis and characterized by NMR and high resolution electrospray ionization mass spectrometry (HR-ESIMS), as previously reported. ${ }^{1}$ Accurate mass measured for each oligomer is listed in Table S1. Dimethyl sulfoxide (DMSO, Alfa Aesar, >99\%) was used as received.

\section{A.2. Crystals preparation}

The general crystallization procedure of digitally-encoded polyurethanes goes as follows. A volume of water was added in a screw neck glass vial containing the same volume of a solution of the desired compound in DMSO $\left(2.5-5 \mathrm{mg} \cdot \mathrm{mL}^{-1}\right)$. The resulting suspension was heated until the total dissolution of the precipitate. The vial was then closed, immerged in an oil bath at $120^{\circ} \mathrm{C}$, and slowly allowed to cool down to room temperature. This general procedure afforded crystal suspensions which were further analyzed.

\section{A.3. Transmission Electron Microscopy}

The oligourethane crystals were characterized by electron diffraction with a Philips CM12 microscope equipped with a digital camera (Megaview III, Soft Imaging Systems). A drop of a crystal suspension was deposed between a glass slide and a cover slip. The resulting sample was first examined by phase contrast microscopy in order to determine crystal microscopic dimensions and ED suitability. A drop of the crystal suspension was then deposed on a carbon coated TEM grid before analysis. For all samples, an internal gold calibration was used. Gold coating was performed by vaporization. 


\section{B. Additional data and figures}

Table S1. Characterization of the digital oligourethanes used in this work.

\begin{tabular}{|c|c|c|c|c|c|}
\hline Sequence $^{\mathrm{a}}$ & $m / z_{\text {th }}{ }^{\mathrm{b}}$ & $m / z_{\exp }{ }^{b}$ & $\mathbf{a}^{\mathbf{c}}(\stackrel{\AA}{\mathbf{A}})$ & $\mathbf{b}^{\mathbf{c}}(\AA)$ & $V_{\text {bit }^{\mathbf{d}}}{ }^{\mathrm{d}}\left(\AA^{3}\right)$ \\
\hline$\alpha 000000$ & 840.4924 & 840.4921 & 4.76 & 3.66 & 148 \\
\hline$\alpha 000001$ & 854.5081 & 854.5080 & 4.79 & 3.67 & 149 \\
\hline$\alpha 010000$ & 854.5081 & 854.5084 & 4.75 & 3.82 & 154 \\
\hline$\alpha 001000$ & 854.5081 & 854.5074 & 4.86 & 3.88 & 160 \\
\hline$\alpha 010010$ & 868.5237 & 868.5242 & 4.85 & 4.04 & 167 \\
\hline$\alpha 010101$ & 882.5394 & 882.5392 & 4.83 & 4.08 & 168 \\
\hline$\alpha 011111$ & 910.5707 & 910.5714 & 4.78 & 4.36 & 177 \\
\hline$\alpha 111110$ & 910.5707 & 910.5714 & 4.82 & 4.47 & 183 \\
\hline$\alpha 111111$ & 924.5863 & 924.586 & 4.88 & 4.53 & 188 \\
\hline
\end{tabular}

${ }^{\text {a }}$ The letter $\alpha$ represents the caproic acid end-group of the oligomer. The numbers 0 and 1 correspond to the different synthons, respectively butyl carbamate (bit 0 ) and methyl-butyl carbamate (bit 1). ${ }^{\mathrm{b}}$ Theoretical and experimental $\mathrm{m} / \mathrm{z}$ values found by HR-ESI-MS for ammonium adducts. ${ }^{\mathrm{c}} \mathbf{a}$ and $\mathbf{b}$ correspond respectively to the distance between oligomer sheets in the hydrogen bond axis and in the inter-sheet axis determined by electron diffraction pattern resolution. $V_{\text {bit }}$ is the calculated volume occupied by one basic information unit in the crystals. 

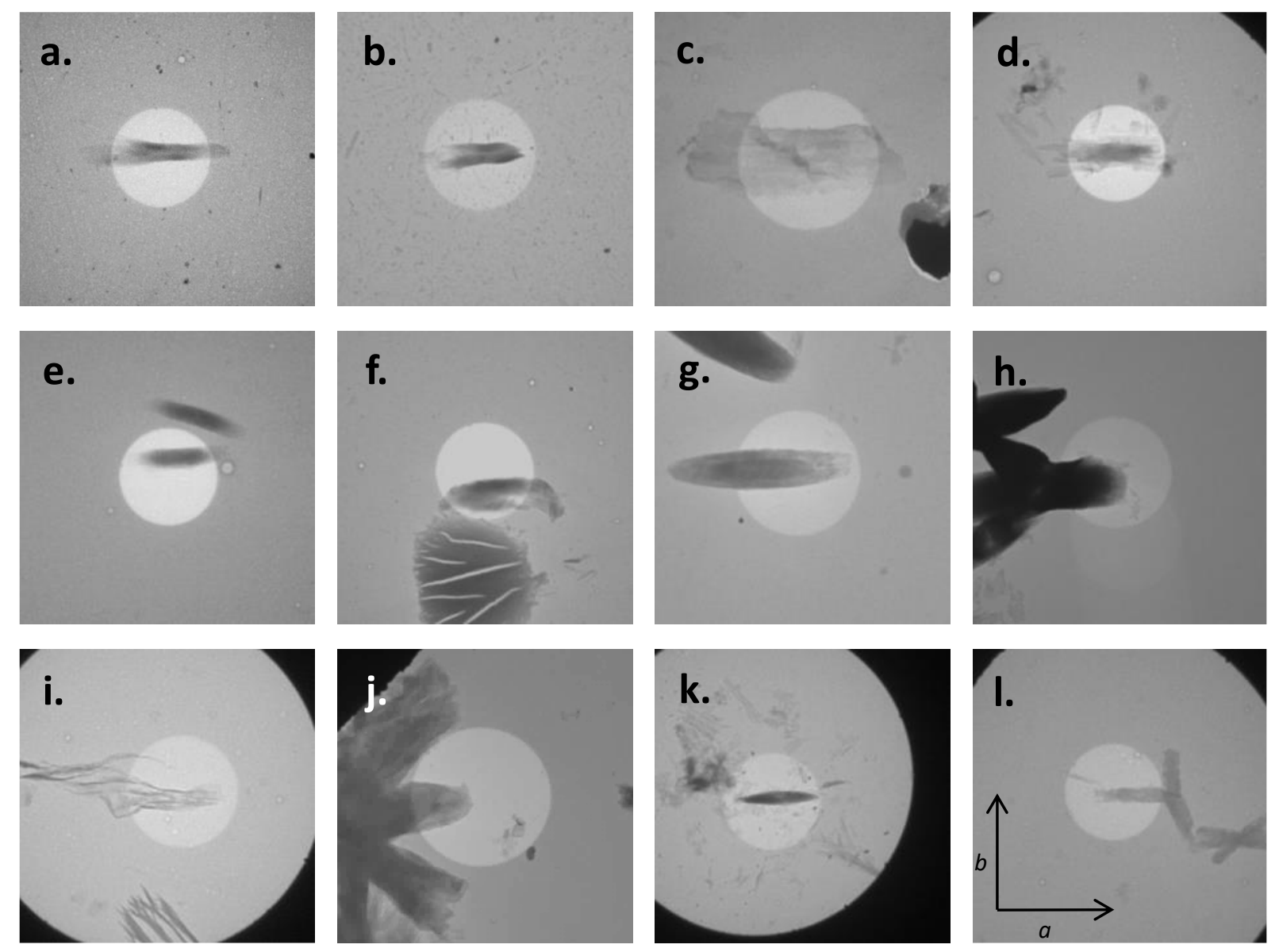

Figure S1. Transmission electron micrographs of the digitally-encoded crystals examined for this work: (a) $\alpha 000000$, (b) $\alpha 000001$, (c) $\alpha 010000$, (d) $\alpha 001000$, (e) $\alpha 010010$, (f) $\alpha 010101$, (g) $\alpha 011111$, (h) $\alpha 111110$, (i) $\alpha 111111$, (j) crystal blend of $\alpha 000000$ and $\alpha 111111$ obtained from a 1:1 mixture, (k) crystal blend of $\alpha 000000$ and $\alpha 111111$ obtained from a 3:1 mixture, (l) crystal blend of $\alpha 000000$ and $\alpha 111111$ obtained from a 1:3 mixture. On each micrograph, the bright circle corresponds to the experimentally irradiated zone affording ED pattern. To highlight similarities between crystal shape and electron diffraction patterns, micrographs were orientated along the $a$ axis. 
a.

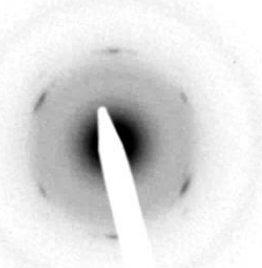

e.

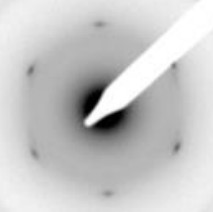

i.

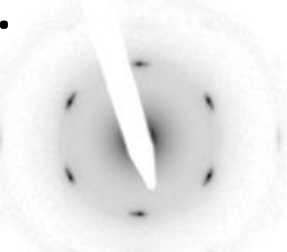

b.

f.

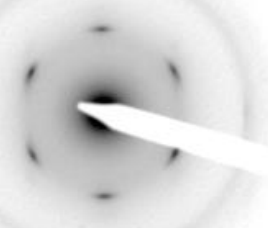

j.

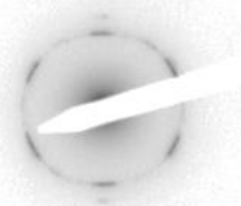

c.

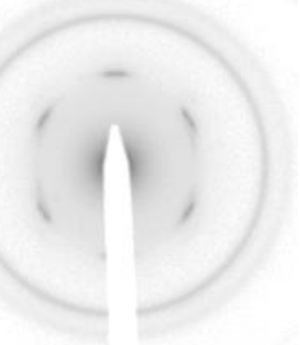

g.

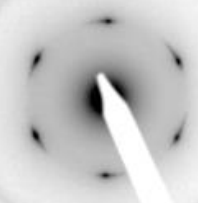

k.

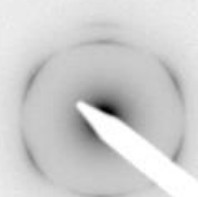

d.

h.

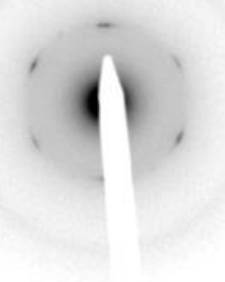

I.

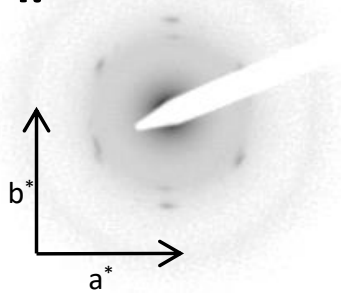

Figure S2. Electron diffraction patterns of the digitally-encoded crystals showed in Figure S1: (a)

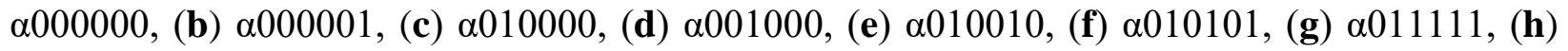
$\alpha 111110$, (i) $\alpha 111111$, (j) crystal blend of $\alpha 000000$ and $\alpha 111111$ obtained from a 1:1 mixture, (k) crystal blend of $\alpha 000000$ and $\alpha 111111$ obtained from a 3:1 mixture, (l) crystal blend of $\alpha 000000$ and $\alpha 111111$ obtained from a 1:3 mixture. To highlight similarities between crystal shape and ED patterns, ED patterns were orientated along the $\mathrm{a}^{*}$ axis. 

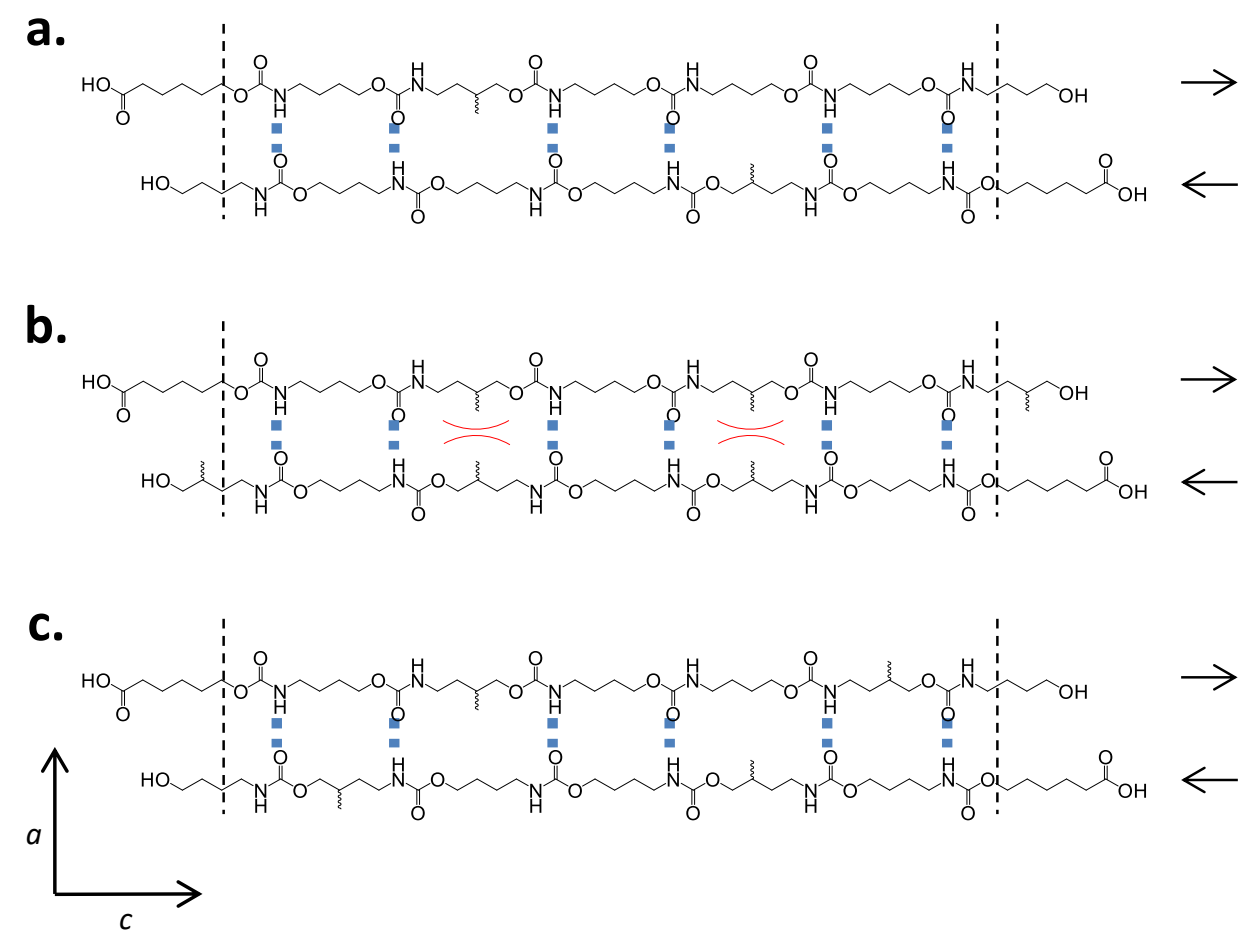

Figure S3: Antiparallel arrangement of the chains for oligomers $\alpha 010000$ (a), $\alpha 010101$ (b) and $\alpha 010010$ (c). Hydrogen bonds and steric hindrances are respectively highlighted by blue dashes and red curves. Lattice in the c axis limits are showed by dashed lines.

\section{References.}

(1) Gunay, U. S.; Petit, B. E.; Karamessini, D.; Al Ouahabi, A.; Amalian, J.-A.; Chendo, C.; Bouquey, M.; Gigmes, D.; Charles, L.; Lutz, J.-F. Chemoselective Synthesis of Uniform Sequence-Coded Polyurethanes and Their Use as Molecular Tags; Chem 2016, 1, 114 